\title{
Clinical Decision Support for the Diagnosis and Management of Adult and Pediatric Hypertension
}

\author{
Suchith Vuppala ${ }^{1} \cdot$ Christy B. Turer ${ }^{2}$ \\ Published online: 27 August 2020 \\ (C) Springer Science+Business Media, LLC, part of Springer Nature 2020
}

\begin{abstract}
Purpose of Review To review literature from 2016 to 2019 on clinical decision support (CDS) for diagnosis and management of hypertension in children and adults.

Recent Findings Ten studies described hypertension CDS systems. Novel advances included the integration of patient-collected blood pressure data, automated information retrieval and management support, and use of CDS in low-resource/developing-world settings and in pediatrics. Findings suggest that CDS increases hypertension detection/control, yet many children and adults with hypertension remain undetected or undercontrolled. CDS challenges included poor usability (from lack of health record integration, excessive data entry requests, and wireless connectivity challenges) and lack of clinician trust in blood pressure measures.

Summary Hypertension CDS has improved but not closed gaps in the detection and control of hypertension in children and adults. The studies reviewed indicate that the usability of CDS and the system where CDS is deployed (e.g., commitment to highquality blood pressure measurement/infrastructure) may impact CDS's ability to increase hypertension detection and control.
\end{abstract}

Keywords Hypertension $\cdot$ High blood pressure $\cdot$ Clinical decision support $\cdot$ Pediatric hypertension $\cdot$ Electronic health record

\section{Introduction}

Hypertension is prevalent and deadly, yet treatable and increasingly observed in youth [1-4]. Over 1.1 billion people worldwide and 103 million people in the USA have high blood pressure (BP) [1,3]. The onset of hypertension in youth is concerning. The disease lacks symptoms, is associated with markers of future cardiovascular (CV) morbidity in childhood, and causes stroke, kidney failure, and heart failure if untreated [2, 5-7]. In adults with hypertension, BP control decreases CV disease and death $[2,8-10]$.

This article is part of the Topical Collection on Implementation to Increase Blood Pressure Control: What Works?

Christy B. Turer

Christy.Turer@UTSouthwestern.edu

Suchith Vuppala

Suchith.Vuppala@UTSouthwestern.edu

1 Department of Medicine, University of Texas Southwestern (UTSW) Medical School, Dallas, TX, USA

2 Departments of Pediatrics and Medicine, UTSW and Children's Health, 5323 Harry Hines Blvd., Dallas, TX 75390-9063, USA
In the $42 \%$ of US adults with hypertension, the disease is recognized by $94 \%$ of providers and $82 \%$ of patients $[2,3]$. BP control, however, is achieved by only $48 \%$ of US adults with hypertension overall and $16 \%$ of men ages $18-39$ years [3]. Sadly, hypertension-related deaths have increased $38 \%$ from 2005 to 2015 despite the availability of effective hypertension therapies [2]. Higher rates of BP control are needed among adults with hypertension.

Hypertension increasingly affects youth due to obesity [4]. Diagnosing hypertension in children is more complex than for adults [7]. In children ages 1-13 years, hypertension diagnosis requires three auscultated BPs at three separate visits $\geq 95$ th percentile for sex, age, and height or $\geq 130 / 80 \mathrm{mmHg}$ (whichever measure is lower) [7]. Whereas $2-5 \%$ of healthy weight children in the USA have hypertension, prevalence among adolescents with severe obesity approaches 14-15\% [4]. Yet the rate of provider recognition of pediatric hypertension is only 20-25\%, and provider communication to families of a child who has high BP is even lower (15\%) [11-13]. Because identifying hypertension in children is complex, there is a great need to improve diagnosis of pediatric hypertension [14•].

A promising tool to increase hypertension diagnosis among children and BP control among adults is clinical decision 
support (CDS) $[15,14 \bullet]$. CDS can detect elevated BP or BPpercentile readings at clinic visits, and support clinicians in making a diagnosis, evaluating secondary causes, providing lifestyle counseling, and using pharmacotherapy. Developing CDS involves converting guidelines into computer-readable logic (for example, programming thresholds for hypertension); mapping workflow, documentation, manual, and cognitive processes through observation and stakeholder engagement; automating cognitively challenging tasks, including computation of BP percentiles for age, sex, and height, and time-consuming manual tasks, including retrieval of BP at past visits; developing a user interface with clinician/staff input and testing, and iterative improvement of the interface design; and updating the system logic as guidelines change [16]. The goal of CDS is to bridge gaps in clinician knowledge of guidelines, circumvent challenges posed by competing priorities (for example, patient concerns vs. health-maintenance items), and reduce variation in healthcare delivery $[15,16]$.

The benchmark for use of CDS to systematically improve hypertension care has been set by Kaiser Permanente. Kaiser Permanente used CDS alongside healthcare delivery redesign to increase BP control for every race/ethnicity from $48 \%$ (the national average) in 2004 to $86 \%$ in 2012 and reduce CV death, defining BP control using the established quality measure (the percentage of adults with a hypertension diagnosis whose BP was < 140/90 mmHg [17]) [18•, 19, 20]. Decades before, the Kaiser Permanente system had already been implementing system-wide periodic staff training on highquality BP measurement, simple treatment algorithms initiating patients with high BP on two antihypertensive medications, and registry tracking of hypertension care with provider performance feedback [20]. A 2000-2004 redesign built on these measures, using CDS crafted from workflow process mapping that suggested a need for information-system integration and self-management support for patients [19]. From 2000 to 2015, deaths from heart disease in the Kaiser Permanente population declined by $48 \%$ and from stroke by 56\% [21]. Kaiser Permanente set the benchmark for achieving high rates of $\mathrm{BP}$ control and reducing major $\mathrm{CV}$ events through systematized hypertension care that included CDS.

This paper critically appraises literature published from 2016 to 2019 regarding pediatric and adult hypertension CDS [14•]. Findings are used to discuss opportunities to improve the value of hypertension CDS to increase the diagnosis and control of high BP in children and adults.

\section{Methods}

The literature review was performed by searching PubMed. The terms "hypertension" and "decision support" were combined. Papers on "pulmonary hypertension," "portal hypertension," and "intracranial hypertension" were excluded.

\section{Inclusion/Exclusion Criteria}

Publication date was limited to Jan 1, 2016, to Dec 31, 2019. Abstracts of the identified papers were reviewed to verify that the study focused on use of CDS to identify/manage elevated BPs or hypertension in children or adults in outpatient settings. Studies meeting inclusion/exclusion criteria were pulled for full-text review.

\section{Full-Text Review and Qualitative Analysis}

The authors (SV, CT) independently reviewed the full-text articles and abstracted data on each study's aim, CDS solution, study design/setting, and outcome(s). Features of each CDS system were described. For example, information was sought regarding how the CDS was delivered (e.g., EHR, web), aspects of hypertension care supported (e.g., repeating elevated BP measures, diagnosis, evaluation, management), and who among the care team was supported (e.g., patient, clinical staff, pharmacists, and providers/physicians). The authors met to compare notes and develop consensus.

\section{Results}

The literature search identified 208 articles. Articles $(n=188)$ were excluded due to lack of focus on essential hypertension $(n=132)$, not CDS $(n=49)$, study setting not outpatient $(n=$ $2)$, literature review only $(n=3)$, or consumer app without a clinician diagnosis/management component $(n=2)$. The final review included 20 full-text articles reporting results from 10 unique studies, including three studies in children $(n=5$ articles) and seven in adults ( $n=15$ articles).

\section{Pediatric Hypertension CDS}

1. The TeenBP study tested a CDS system designed to aid pediatrician diagnosis and management of hypertension in 10-17-year-olds $[22 \bullet, 23]$. On patient check-in, TeenBP alerted clinic staff to collect height if no height was entered (because height is needed to compute BP percentiles in $<13$-year-olds) and to remeasure $\mathrm{BP}$ when high [24]. TeenBP alerted clinicians to a patient's high BP and offered an EHR-linked, web-based interface to view the patient's BP history, medical history, risk factors (e.g., high body mass index [BMI], medications that increase BP), and guideline-recommended management options (refer to a dietitian, evaluate secondary causes of hypertension, follow up at a guideline-based interval, and print patient-education materials).

To test TeenBP, 20 Midwestern US primary care practices were randomized to usual care or hypertension CDS [23]. Hypertension recognition, diagnosis, and follow-up 
were defined using visit-associated diagnosis codes. Among 10-17-year-olds screened over 1 year $(n=$ 21,648 ), pediatricians at sites with CDS had higher rates of recognizing an incident hypertensive BP (28\% of 607 teens with a hypertensive BP in the CDS arm, vs. $4 \%$ of 607 in the control arm; $P<.001)$ [22 $]$. After 2 years $(n=$ 31,579 screened), greater proportions of 10-17-year-olds with a third incident hypertensive BP received a hypertension diagnosis (55\% CDS, $21 \%$ control; $P \leq 0.001$ ) [23]; however, follow-up to remeasure BP within 30 days did not differ between groups (14\% CDS, $11 \%$ control).

2. The Crete CV Screening tool, developed in Greece, provided EHR-integrated support for detecting high BP and CV disease risk factors in children [25]. The tool alerted staff to repeat an elevated BP measure, computed BP percentiles, and used a risk algorithm to support cardiologists in identifying a child needing cardiac work-up. The risk algorithm incorporated information about a child's family/medical history, BP, BMI, and cardiac studies. Detection of high BP was a small component of a larger $\mathrm{CV}$-screening system and investigators reported data on the tool's usability. No data were reported regarding the effectiveness of the tool for detecting high BP or other CV disease risk factors in children.

3. The final pediatric study reviewed presented data on the impact of the SMART BP Centiles app on recognition of elevated ( $>90$ th centile) BPs at clinics in Boston, MA [26]. When a provider clicked on an EHR-embedded link to the app, a child's BP percentiles at the visit and at historic visits were displayed (without recommendations for management).

Retrospective EHR data and a pre-poststudy design were used to examine rates of elevated-BP recognition before vs. after app implementation, and among those who clicked on the app [26]. Elevated-BP recognition was defined using diagnosis codes, referrals to nephrology/cardiology, echocardiogram/renal-ultrasound studies, and BP-monitoring follow-up visits. Clinicians accessed the app at $13 \%$ of visits $(N=447 / 3358)$. Recognition of elevated BP was $4.9 \%$ before app integration vs. $7.1 \%$ afterwards $(P<0.01)$, and $16.6 \%$ among clinicians who clicked on the app.

\section{Adult Hypertension CDS}

1. The Empower-H (engaging and motivating patients online with enhanced resources - hypertension) trial was conducted at two clinics in Palo Alto, California (Table 1) [27•]. The focal point of Empower-H was a dashboard that was viewable to both the primary-care team (through the EHR) and to the patient (via the web). The dashboard presented a patient's action/treatment plan,
BPs measured in-office and at home, and step-counts.

A 6-month pre-post (uncontrolled) trial was used to test if the Empower-H system improved BP control (defined as achievement of an in-office $\mathrm{BP}<140 /<$ $90 \mathrm{mmHg}$ ) among adults with resistant, uncontrolled hypertension [27•]. Only eligible participants who completed at least one nurse visit and submitted home BP measurements were enrolled $(n=149)$.

The average participant BP was 150/91 $\mathrm{mmHg}$ at enrollment. At 6 months, 56\% ( $n=143,96 \%$ retention) of participants achieved an average in-office BP $<140 /<90$ and $71 \%$ achieved average home BPs $<135 /<85$. Investigators cited the reduced need for office visits as potentially contributing to the high retention rate and trial success.

2. The mPower Heart project sought to improve identification of hypertension and diabetes in rural community health centers in India using a smartphone-enabled, nurse coordinator-led CDS tool (Table 1)[28•, 29]. To develop mPower, investigators documented barriers to providing guideline-based hypertension/diabetes care such as a high patient-to-physician ratio [28 $]$. Next, the investigators mapped existing health-center workflows and recordkeeping processes. Findings were used to build and refine a prototype and CDS software that underwent cycles of user-acceptance testing, case-study evaluation (on accuracy of CDS plans), and field testing. Then, investigators engaged government officials to determine clinics with sufficient infrastructure (e.g., mobile connectivity/streets) to support a trial and to get buy-in from the health centers.

mPower included use of mobile-enabled CDS with task-shifting among nurses and physicians (to address the high patient-to-physician ratio) [28•,29]. The app generated a tailored management plan using patient data (on demographics, medical history, medications, and BP) entered by a nurse while screening and examining a patient. The nurse handed a printed paper ticket with the CDS plan to a physician for review. The physician accepted the plan or recommended specific modifications to the nurse who then communicated the plan to the patientboth medication therapies and counseling (regarding diet/physical activity and alcohol/tobacco/drug cessation).

The mPower platform was tested in an uncontrolled 18-month observational study at five community health centers [28•]. Each health center was staffed by $3-4$ physicians who collectively cared for up to 200 patients/day. The primary hypertension outcomes were the (a) number of new screen-detected cases of hypertension and (b) mean change in systolic BP (SBP) at 18 months. A secondary outcome was proportion of CDS plans accepted by physicians without modification.

Of 22,009 $\geq 30$-year-olds opportunistically screened, $27 \%$ were identified as having hypertension; $52 \%$ of 
diagnoses were new. SBP decreased by $15 \mathrm{mmHg}$ from baseline; however, only $13 \%$ of participants completed 18-month follow-up measures. The proportion of CDS plans accepted without modification by physicians was $73 \%$. The primary reason physicians cited for changing the plan was lack of medication supply at their clinic. The investigators published study protocols for a randomized controlled trial (RCT) underway at the time of this review $[30,31]$.

3. HIPS (hiding in plain sight) was a prospective, uncontrolled quality-improvement project to improve the rate of diagnosed hypertension using interdisciplinary teams at 10 safety-net health-center organizations across five US states (Table 1) [32•]. Sites used an algorithm that discovered cases of potentially undiagnosed hypertension, defined as no hypertension diagnosis code linked to a visit among adults meeting criteria for stage- 1 hypertension at two medical visits or stage-2 hypertension at one visit in the past year [33]. Some sites embedded the algorithm into the EHR so that a care team received an alert when a patient with undiagnosed hypertension presented for care. Most sites used registries to track/report BP, visits, and diagnoses on patients flagged by the algorithm. Additional measures clinics used to improve hypertension diagnosis included outreach to schedule visits and provision of free BP-check visits.

HIPS aimed to increase the proportion of adults who received a hypertension diagnosis (per a visit-encounterlinked ICD-9 code) and completed a follow-up visit, among all adults flagged with potentially "undiagnosed hypertension" during the 18-month project [32]. After 18 months, $21 \%$ of flagged patients received hypertension diagnoses and 44\% completed follow-up visits. Despite these successes, $79 \%$ of people flagged with potentially undiagnosed hypertension lacked diagnoses at the project's end. The authors cited lack of provider trust in office-based BP measures as a potential reason that more patients did not receive diagnoses.

4. The HOPE-4 (heart outcomes prevention and evaluation 4) study aimed to improve community-based screening of $\mathrm{CV}$ risk factors (including hypertension) in Malaysia and Columbia [34, 35•]. A 12-month RCT was used to test the effectiveness of a system-level intervention that included tablet-based CDS vs. screening without system-level intervention/CDS (control) for improving $\mathrm{CV}$ risk (including BP). Both arms of the trial employed community health workers to perform community-based screenings (Table 1).

In communities randomized to the HOPE-4 intervention $(N=14)$, health workers were guided by tablet-based CDS to obtain/enter medical history, lifestyle behaviors, and $\mathrm{CV}$ risk factors $[34,35 \bullet$. Treatment algorithms guided the health workers in counseling people with CV risk factors (e.g., hypertension), providing free antihypertensive and lipid-lowering medications when indicated (overseen by physicians), and helping participants appoint a support person to aid medication-taking and healthcare follow-up. In control communities $(N=16)$, health workers obtained patient medical/lifestyle history without CDS. After BP and laboratories were obtained, health workers delivered traditional risk communication, health literature, and a recommendation to seek care.

The HOPE-4 trial's primary outcome, the intergroup difference in $\mathrm{CV}$ risk score at 12 months, favored HOPE-4 (11\%, HOPE-4, vs. 6\%, controls, $P>.01$ ) [34]. Prespecified hypertension outcomes also were favorable. At 12 months, 39\% more HOPE-4 participants achieved an SBP $<140 \mathrm{mmHg}(69 \%$, HOPE-4, vs. 30\%, controls; $P<0.01$ ), and the intergroup difference in SBP reduction was $-11 \mathrm{mmHg}(-21 \mathrm{mmHg}$, HOPE-4, vs. $-10 \mathrm{mmHg}$, controls; $P<0.01)$.

5. The mWellcare study shared many notable similarities and differences with the mPower study [36-39, 40•]. Similarities included mWellcare's development by many of the same mPower investigators, a focus on hypertension and diabetes, performance of studies at rural health centers in India, use of task-shifting, a web-based interface to store and provide remote access to patient data, tablets for data entry, and tablet-based CDS (Table 1).

Important differences in the mWellcare study included: performance of an RCT wherein both arms (enhancedusual care and intervention) used tablets for structured data entry, use of rural health centers in northern and southern India, a focus on improving management of (vs. screening for) hypertension/diabetes, and assessment of outcomes at home visits rather than at health centers [36-39]. Nurses and physicians at both enhanced usual care and intervention sites received intensive training on guideline-based BP management, health education pamphlets for distribution to patients, greater access to prescription medications, and follow-up using techniciancoordinated SMS-text messaging. The main difference in the enhanced-usual care arm was that the nurse used a laminated card with guideline-based treatment recommendations to generate a plan. In both arms, the plan was handed to a physician for review, the physician accepted or modified the plan, and a nurse delivered the plan to the patient. Finally, in contrast to mPower's use of existing workflows to drive development of CDS, $\mathrm{mWellcare}$ required changing workflows and staff retraining.

Pilot tests revealed some usability challenges [38]. Physicians reported difficulty using the CDS print-out's medication recommendations. The print-out recommended prescribing a medication within a specific drug class, which meant that physicians had to identify a name of a 
medication in the recommended drug class, and that also was available at the clinic. Pilot test results revealed that physicians accepted the hypertension plan $61 \%$ of the time.

The mWellcare system was tested in a cluster RCT against enhanced usual care for improving BP in patients with hypertension (and blood sugar in patients with diabetes) [40•]. In the RCT, SBP decreased $14 \mathrm{mmHg}$ in the mWellcare arm and $13 \mathrm{mmHg}$ in the control arm, with no significant intergroup difference in SBP $\left(P_{\text {unadjusted }}=\right.$ $0.61)$. Adjustment for baseline intergroup differences in SBP (4 mmHg higher in the control group, $157 \mathrm{vs}$. $153 \mathrm{mmHg}$ ) and greater proportions educated and employed among usual-care participants did not account for the lack of intergroup difference in SBP $\left(P_{\text {adjusted }}=\right.$ $0.9)$. The reason for the lack of intergroup difference in BP cited by the investigators was that all sites offered better systems of care-all sites benefited from use of tablets to enter structured data, increased access to medications, text-facilitated follow-up reminders, and outcomes assessment in the community.

6. Hydra study investigators reported the creation and use (with case studies) of a web-based CDS platform designed to process data from many $\mathrm{CV}$ studies to aid clinicians in the screening/management of $\mathrm{CV}$ diseases in adults, including hypertension [41]. Hydra was developed in Spain and studied for its ability to refine CV risk-stratification-not for improving hypertension detection/ control (results not included in Table 1).

Hydra's logic supported processing data from a wide array of studies, including ambulatory BP monitoring, echocardiograms, EKGs, Holter monitoring, carotid intima-media thickness, pulse-wave velocity, and anklebrachial index [41]. These data and ancillary patient information required manual entry and upload, however, because Hydra lacked integration with an EHR. Once data were entered, Hydra processed the information to present risk stratification and treatment recommendation tables to clinicians for decision-making.

7. TeleHas investigators published results from a mixed methods study that tested the feasibility and usability of a CDS system to improve hypertension care in primary care settings in Brazil [42•]. No patient/pilot data are reported (results not included in Table 1).

TeleHas CDS was accessible to clinicians on tablets [42•]. The system required that clinicians enter patient data (e.g., name, birth date, medical history, risk factors, medications, physical examination, and laboratory studies) and send the data to a central database by establishing an internet connection. TeleHas processed data centrally and pushed recommendations back to the clinician. Recommendations included prescription medication changes and lifestyle modification plans.
TeleHas was tested by 10 primary care physicians who were asked to use the system for 6 months with no prior training, then interviewed/surveyed regarding TeleHas's feasibility/usability at 3 and 6 months [42•]. Physicians reported training would be "essential" for TeleHas use, rated the system's intuitiveness as poor, cited data entry as a major burden, and stated that use of TeleHas delayed their daily routine. Key usability challenges included that the app was unstable, suddenly closed during use, and poor wireless connectivity prevented the ability to upload data. Despite these challenges, the physicians rated TeleHas's treatment recommendations as "good" overall with potential to "improve the treatment of hypertensive patients."

\section{Discussion}

Pediatric and adult hypertension CDS improved hypertension diagnosis in children and both hypertension diagnosis and BP control in adults in many of the contemporary studies reviewed. For pediatric hypertension diagnosis, TeenBP's investigators set a new benchmark, achieving a rate $55 \%$ hypertension diagnosis in the CDS arm (a 34\% higher rate of diagnosis than at sites without CDS) [23]. For BP control in adults, however, no trial bettered Kaiser Permanente's 86\% BP control among covered individuals (across all race/ethnicities) [19]. Of the pediatric and adult hypertension CDS trials reviewed, many shared challenges with retaining patients/ ensuring follow-up to remeasure BP, lack of clinician trust in BP measures, and usability.

The two pediatric hypertension CDS systems that reported trial results differed in the amount of diagnostic and management decision support provided to clinicians and in their impact on elevated BP recognition/hypertension diagnosis. The SMART BP-Centiles app supported clinicians in viewing visit-/historic-BP percentiles only, and increased elevated BP recognition by $\sim 12 \%$ (from 4.9 to $16.6 \%$ ) [26]. TeenBP presented both the BP-percentile data and guideline-based options for hypertension diagnosis and management, and increased clinician recognition of an incident high BP by $24 \%$ (to 28\%), compared with no $\operatorname{CDS}[22 \bullet, 23]$. Thus, robust diagnostic/management CDS may be needed to improve recognition of high BPs in children.

Two adult hypertension CDS trials incorporated BP information obtained outside of office settings - a critical feature needed in the post COVID-19 era [43]. Empower-H used a mobile interface to integrate BPs obtained at home into an EHR, then processed/presented information to both the patient and care team [27•]. In contrast, although Hydra was able to process ambulatory BP monitoring data (and additional $\mathrm{CV}$ 


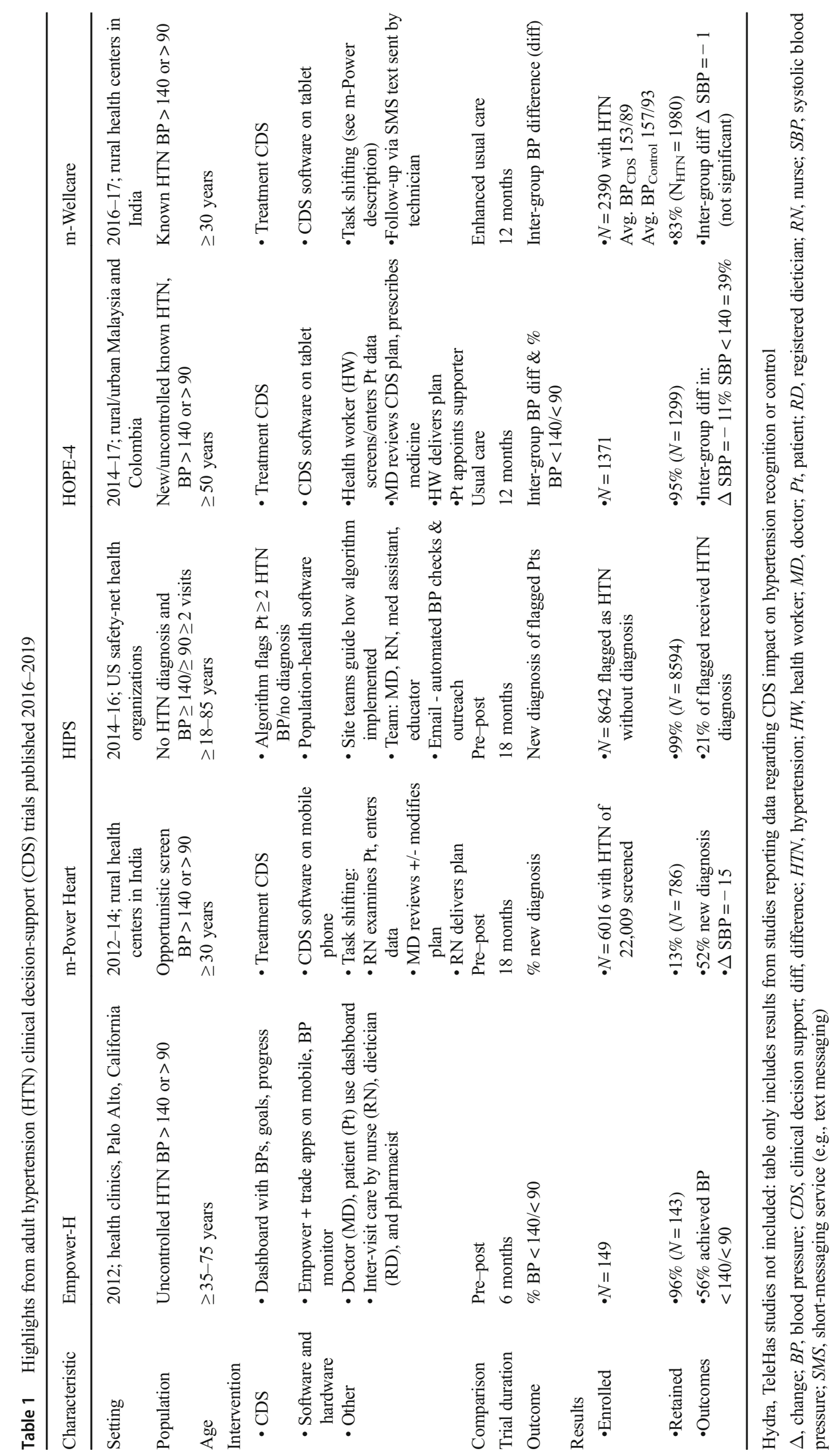


studies), the system required manual data entry [41]. Thus, Empower-H (which automated collection/presentation of BP data) succeeded where Hydra (with its repeated requests for data input and usability failures) failed; results caution those building CDS to limit requests for data input - a cardinal rule of effective CDS design [16].

CDS was used to reach low-resource settings in the mPower, HOPE-4, mWellcare, TeleHas, and HIPS studies; however, only HOPE-4 and mPower reported significantly greater BP improvements with CDS. HIPS used site-specific application of an algorithm to diagnose $21 \%$ of previously undiagnosed hypertension cases, citing poor provider trust in $\mathrm{BP}$ measures as the reason that more cases were not diagnosed [32•]. TeleHas suffered from poor usability and wireless connectivity problems[42•], whereas, mWellcare improved BP control in both the CDS and control arms - which investigators attributed to systemwide improvements in hypertension care that took place at the same time as the trial. Yet, control groups differed vastly between the null mWellcare and the successful HOPE-4 RCTs. Whereas mWellcare armed both $\mathrm{CDS} /$ control arms with tablet-enabled structured data entry, HOPE-4's health workers obtained patient histories per usual care (without tablets). Similarly, whereas nurses in mWellcare's control arm used laminated algorithms to construct hypertension plans for physician review, HOPE-4's control health workers delivered generic risk communication, health literature, and a recommendation to seek care. In sum, results suggest that the most important features of effective hypertension care in low-resource settings include systemwide hypertension-care improvements with high-quality BP measurement, structured data entry, algorithm-based treatment planning, task-shifting (among nurses/physicians), and paying special attention to ensuring follow-up.

\section{Future Directions}

A key challenge encountered in both the pediatric and adult trials of hypertension CDS was the lack of provider trust in clinic-obtained BP values. Yet, adult and pediatric health systems handle this lack of trust in BP differently due to reforms to improve the quality of care enacted through the affordable care account, accountable care rules, and value-based payment initiatives [44, 45]. The percent of adults with hypertension achieving BP control is an established quality metric [17]. Thus, health systems accountable for the health of adult populations are financially incentivized to ensure confidence in BP measures. Value-based payment initiatives drive system level solutions, including periodic staff retraining on BP measurement, use of registry-collected data for provider performance feedback, and use of hypertension CDS [17].

There is no comparable quality metric in children to prompt diagnosis of pediatric hypertension or to build pediatric hypertension CDS, because of an absence of data that treating hypertension in childhood reduces adverse health outcomes associated with hypertension in adults [46]; this absence of data should not be misconstrued as the presence of data suggesting an absence of effect. Compounding the vacuum of longitudinal-outcomes data is the complexity needed to make a guideline-based pediatric hypertension diagnosis. Guidelines recommend that clinicians (1) auscultate BP (auscultating BP in a child yields an SBP that is $1.8-2.5 \mathrm{mmHg}$ higher than oscillometric BP with no difference in DBP [47]); (2) use the average of three auscultated BPs to determine if the average visit BP exceeds the BP-percentile threshold for age, sex, and height: and ( 3 ) verify that the child meets the definition of pediatric hypertension per auscultated BPs at $\geq 3$ separate visits [7]. The far less complex adult definition of hypertension (use oscillometric or auscultated BP to identify BP elevations at $\geq 2$ visits [8]) is bolstered by data that $\mathrm{BP}$ control prevents adverse health consequences [8, 10, 17]. CDS cannot resolve BP measurement challenges, and systematically low rates of provider detection of high BP in children limit use of EHR data to answer whether treating hypertension in children reduces adverse health outcomes in adults. Without a pediatric hypertension quality metric, health systems lack the incentives needed to build pediatric hypertension CDS, to prompt clinics to track pediatric hypertension care, and to motivate pediatricians to diagnose hypertension [17].

In adults, the complexity of hypertension care lies in a patient's medical history, medications, renal function, and both desire and ability to access healthcare and prescription medications. In the CDS systems reviewed, only Crete CV and Hydra incorporated information such as left ventricular hypertrophy on EKG or creatinine elevation indicative of chronic kidney disease into decision-making. No system provided information about patient adherence to prescription antihypertensives. Effective CDS automates the processing/provision of information that exacts a high cognitive burden from a clinician: for example, presenting EKG-findings of left ventricular hypertrophy (LVH) when a clinician is considering if an elevated BP represents true vs. white-coat hypertension, or presenting a medication-adherence score based on antihypertensive-medication-refill patterns. Well-designed user-centered CDS holds great promise for reintegrating scattered health information so clinicians can provide the highest quality care to patients [16].

\section{Conclusions}

The contemporary studies reviewed indicate that hypertension CDS may improve rates of hypertension diagnosis in children and BP control in adults. To address barriers to achieving higher levels of diagnosis and BP control, the studies indicate that governments and health systems may prove critical for securing wireless infrastructure to support rapid data transfer 
and the high-level commitment needed to ensure durable high-quality BP measurement. In children, CDS dissemination may require a BP quality metric. To aid clinical decision-making in the care of complex adults with hypertension, future CDS systems may by improved through processing/presenting $\mathrm{CV}$-study data residing within the EHR (e.g., LVH), from home BP-monitoring data, and through linkage to pharmacy systems.

\section{Compliance with Ethical Standards}

Conflict of Interest The authors declare that they have no conflict of interest.

Human and Animal Rights and Informed Consent This article does not contain any studies with human or animal subjects performed by any of the authors.

\section{References}

Papers of particular interest, published recently, have been highlighted as:

- Of importance

1. World Health Organization. Hypertension. https://www.who.int/ news-room/fact-sheets/detail/hypertension. Accessed May 72020.

2. Benjamin EJ, Muntner P, Alonso A, Bittencourt MS, Callaway CW, Carson AP, et al. Heart disease and stroke statistics-2019 update: a report from the American Heart Association. Circulation. 2019;139(10):e56-e528. https://doi.org/10.1161/CIR. 0000000000000659 .

3. Fryar CD, Ostchega Y, Hales CM, Zhang G, Kruszon-Moran D. Hypertension prevalence and control among adults: United States, 2015-2016. NCHS Data Brief 2017(289):1-8.

4. Jackson SL, Zhang Z, Wiltz JL, Loustalot F, Ritchey MD, Goodman AB et al. Hypertension among youths - United States, 2001-2016. MMWR Morbidity and Mortality Weekly Report. 2018;67(27):758-62. doi:https://doi.org/10.15585/mmwr. mm6727a2.

5. Brady TM, Appel LJ, Holmes KW, Fivush B, Miller ER, 3rd. Association between adiposity and left ventricular mass in children with hypertension. J Clin Hyperten (Greenwich, Conn). 2016;18(7):625-33. doi:https://doi.org/10.1111/jch.12717.

6. Daniels SR. Adverse outcomes in pediatric hypertension. Hypertension. 2017;70(6):1089-90. https://doi.org/10.1161/ HYPERTENSIONAHA.117.09784.

7. Flynn JT, Kaelber DC, Baker-Smith CM, Blowey D, Carroll AE, Daniels SR et al. Clinical practice guideline for screening and management of high blood pressure in children and adolescents. Pediatrics. 2017;140(3). doi:https://doi.org/10.1542/peds.20171904.

8. Carey RM, Whelton PK, Committee AAHGW. Prevention, detection, evaluation, and management of high blood pressure in adults: synopsis of the 2017 American College of Cardiology/American Heart Association hypertension guideline. Ann Intern Med. 2018;168(5):351-8. https://doi.org/10.7326/M17-3203.

9. Okin PM, Devereux RB, Jern S, Kjeldsen SE, Julius S, Nieminen MS, et al. Regression of electrocardiographic left ventricular hypertrophy during antihypertensive treatment and the prediction of major cardiovascular events. JAMA. 2004;292(19):2343-9. https://doi.org/10.1001/jama.292.19.2343.

10. Saklayen MG, Deshpande NV. Timeline of history of hypertension treatment. Front Cardiovasc Med. 2016;3:3. https://doi.org/10. 3389/fcvm.2016.00003.

11. Bismar N, Barlow SE, Brady TM, Turer CB. Pediatrician communication about high blood pressure in children with overweight/ obesity during well-child visits. Acad Pediatr. 2019. https://doi. org/10.1016/j.acap.2019.11.012.

12. Brady TM. Poor elevated-blood-pressure recognition in the outpatient setting. J Pediatr. 2015;167(3):780-1. https://doi.org/10.1016/ j.jpeds.2015.06.060.

13. Hansen ML, Gunn PW, Kaelber DC. Underdiagnosis of hypertension in children and adolescents. JAMA. 2007;298(8):874-9. https://doi.org/10.1001/jama.298.8.874.

14. Semanik MG. The use of electronic health records to identify children with elevated blood pressure and hypertension. Curr Hyperten Rep. 2017;19(12):98. doi:https://doi.org/10.1007/s11906-0170794-2. Past review by this journal on use of CDS to improve pediatric hypertension diagnosis and management.

15. Goertzel G. Clinical decision support system. Ann N Y Acad Sci. 1969;161(2):689-93. https://doi.org/10.1111/j.1749-6632.1969. tb34100.x.

16. Bates DW, Kuperman GJ, Wang S, Gandhi T, Kittler A, Volk L, et al. Ten commandments for effective clinical decision support: making the practice of evidence-based medicine a reality. J Am Med Inform Assoc. 2003;10(6):523-30. https://doi.org/10.1197/ jamia.M1370.

17. Sennett C. Implementing the new HEDIS hypertension performance measure. Manag Care. 2000;9(4 Suppl):2-17 quiz 8-21.

18. Kanter MH, Lindsay G, Bellows J, Chase A. Complete care at Kaiser Permanente: transforming chronic and preventive care. Jt Comm J Qual Patient Saf. 2013;39(11):484-94. https://doi.org/10. 1016/s1553-7250(13)39064-3. Highlights benchmark set in BP control among adults with hypertension.

19. Shaw KM, Handler J, Wall HK, Kanter MH. Improving blood pressure control in a large multiethnic California population through changes in health care delivery, 2004-2012. Prev Chronic Dis. 2014;11:E191. https://doi.org/10.5888/pcd11.140173.

20. Sim JJ, Handler J, Jacobsen SJ, Kanter MH. Systemic implementation strategies to improve hypertension: the Kaiser Permanente Southern California experience. The Canadian Journal of Cardiology. 2014;30(5):544-52. https://doi.org/10.1016/j.cjca. 2014.01.003.

21. Sidney S, Sorel ME, Quesenberry CP, Jaffe MG, Solomon MD, Nguyen-Huynh MN, et al. Comparative trends in heart disease, stroke, and all-cause mortality in the United States and a large integrated healthcare delivery system. Am J Med. 2018;131(7): 829-36 e1. https://doi.org/10.1016/j.amjmed.2018.02.014.

22. Kharbanda EO, Asche SE, Sinaiko A, Nordin JD, Ekstrom HL, Fontaine $\mathrm{P}$ et al. Evaluation of an electronic clinical decision support tool for incident elevated BP in adolescents. Acad Pediatr. 2017. https://doi.org/10.1016/j.acap.2017.07.004. Study that set the benchmark in pediatric hypertension CDS for improving rate of pediatric hypertension diagnosis to $55 \%$ in CDS arm.

23. Kharbanda EO, Asche SE, Sinaiko AR, Ekstrom HL, Nordin JD, Sherwood NE, et al. Clinical decision support for recognition and management of hypertension: a randomized trial. Pediatrics. 2018;141:e20172954. https://doi.org/10.1542/peds.2017-2954.

24. Kharbanda EO, Nordin JD, Sinaiko AR, Ekstrom HL, Stultz JM, Sherwood NE et al. TeenBP: development and piloting of an EHRlinked clinical decision support system to improve recognition of hypertension in adolescents. EGEMS (Wash DC). 2015;3(2):1142. doi:https://doi.org/10.13063/2327-9214.1142.

25. Chatzakis I, Vassilakis K, Lionis C, Germanakis I. Electronic health record with computerized decision support tools for the purposes of 
a pediatric cardiovascular heart disease screening program in Crete. Comput Methods Prog Biomed. 2018;159:159-66. https://doi.org/ 10.1016/j.cmpb.2018.03.009.

26. Twichell SA, Rea CJ, Melvin P, Capraro AJ, Mandel JC, Ferguson MA, et al. The effect of an electronic health record-based tool on abnormal pediatric blood pressure recognition. Congenit Heart Dis. 2017;12(4):484-90. https://doi.org/10.1111/chd.12469.

27. Lv N, Xiao L, Simmons ML, Rosas LG, Chan A, Entwistle M. Personalized hypertension management using patient-generated health data integrated with electronic health records (EMPOWER-H): six-month pre-post study. J Med Internet Res. 2017;19(9):e311. doi:https://doi.org/10.2196/jmir.7831. Highlights the impact of integrated ambulatory BP data in adult hypertension management.

28. Ajay VS, Jindal D, Roy A, Venugopal V, Sharma R, Pawar A et al. Development of a smartphone-enabled hypertension and diabetes mellitus management package to facilitate evidence-based care delivery in primary healthcare facilities in India: the mPower Heart Project. J Am Heart Assoc. 2016;5(12). https://doi.org/10.1161/ JAHA.116.004343. Reports on smartphone-enabled, nursecoordinator led adult HTN CDS tool in low-resource setting in India.

29. Jindal D, Roy A, Ajay VS, Yadav SK, Prabhakaran D, Tandon N. Strategies for stakeholder engagement and uptake of new intervention: experience from state-wide implementation of mHealth technology for NCD care in Tripura. India Glob Heart. 2019;14(2):16572. https://doi.org/10.1016/j.gheart.2019.06.002.

30. Srinivasapura Venkateshmurthy N, Ajay VS, Mohan S, Jindal D, Anand S, Kondal D, et al. M-power heart project - a nurse care coordinator led, mHealth enabled intervention to improve the management of hypertension in India: study protocol for a cluster randomized trial. Trials. 2018;19(1):429. https://doi.org/10.1186/ s13063-018-2813-2.

31. Srinivasapura Venkateshmurthy N, Mc Namara K, Koorts H, Mohan S, V SA, Jindal D et al. Process evaluation protocol for a cluster randomised trial of a complex, nurse-led intervention to improve hypertension management in India. BMJ Open. 2019;9(5):e027841. doi:https://doi.org/10.1136/bmjopen-2018027841.

32. Meador M, Osheroff JA, Reisler B. Improving identification and diagnosis of hypertensive patients hiding in plain sight (HIPS) in health centers. Jt Comm J Qual Patient Saf. 2018;44(3):117-29. doi:https://doi.org/10.1016/j.jcjq.2017.09.003. Highlights the use of algorithm-driven identification of patients with undiagnosed hypertension.

33. Centers for Medicare \& Medicaid Services. ICD-9-CM diagnosis and procedure codes: abbreviated and full code titles, version 32 . Effective 1 Oct 2014. www.cms.gov/Medicare/Coding/ ICD9ProviderDiagnosticCodes/codes.html.

34. Schwalm JD, McCready T, Lopez-Jaramillo P, Yusoff K, Attaran A, Lamelas $\mathrm{P}$ et al. A community-based comprehensive intervention to reduce cardiovascular risk in hypertension (HOPE 4): a clusterrandomised controlled trial. Lancet (London, England). 2019;394(10205):1231-42. https://doi.org/10.1016/S01406736(19)31949-X.

35. Schwalm JR, McCready T, Lamelas P, Musa H, Lopez-Jaramillo P, Yusoff $\mathrm{K}$ et al. Rationale and design of a cluster randomized trial of a multifaceted intervention in people with hypertension: The Heart Outcomes Prevention and Evaluation 4 (HOPE-4) Study. Am Heart J. 2018;203:57-66. https://doi.org/10.1016/j.ahj.2018.06.004. Multinational adult HTN RCT with intervention arm (system level-intervention and CDS) vs control arm (no system level intervention or CDS).

36. Anand S, Patil RB, Krishnamoorthy P. An analytics based clinical decision support system for CVD risk assessment and management.
Conference Proceedings: Annual International Conference of the IEEE Engineering in Medicine and Biology Society IEEE Engineering in Medicine and Biology Society Annual Conference 2016;2016:5620-5623. doi:https://doi.org/10.1109/EMBC.2016. 7592001.

37. Jha D, Gupta P, Ajay VS, Jindal D, Perel P, Prieto-Merino D, et al. Protocol for the mWellcare trial: a multicentre, cluster randomised, 12-month, controlled trial to compare the effectiveness of mWellcare, an mHealth system for an integrated management of patients with hypertension and diabetes, versus enhanced usual care in India. BMJ Open. 2017;7(8):e014851. https://doi.org/10.1136/ bmjopen-2016-014851.

38. Jindal D, Gupta P, Jha D, Ajay VS, Goenka S, Jacob P, et al. Development of mWellcare: an mHealth intervention for integrated management of hypertension and diabetes in low-resource settings. Glob Health Action. 2018;11(1):1517930. https://doi.org/10.1080/ 16549716.2018.1517930.

39. Jindal D, Gupta P, Jha D, Ajay VS, Jacob P, Mehrotra K, et al. The development of mWellcare, an mHealth system for the integrated management of hypertension and diabetes in primary care. Stud Health Technol Inform. 2017;245:1230.

40. Prabhakaran D, Jha D, Prieto-Merino D, Roy A, Singh K, Ajay VS et al. Effectiveness of an mHealth-based electronic decision support system for integrated management of chronic conditions in primary care: The mWellcare cluster-randomized controlled trial. Circulation. 2018. https://doi.org/10.1161/CIRCULATIONAHA. 118.038192. RCT on adult HTN CDS in low-resource setting.

41. Novo J, Hermida A, Ortega M, Barreira N, Penedo MG, Lopez JE, et al. Hydra: a web-based system for cardiovascular analysis, diagnosis and treatment. Comput Methods Prog Biomed. 2017;139:6181. https://doi.org/10.1016/j.cmpb.2016.10.019.

42. Silveira DV, Marcolino MS, Machado EL, Ferreira CG, Alkmim MBM, Resende ES et al. Development and evaluation of a mobile decision support system for hypertension management in the primary care setting in Brazil: Mixed-methods field study on usability, feasibility, and utility. JMIR Mhealth Uhealth. 2019;7(3):e9869. https://doi.org/10.2196/mhealth.9869. Adult CDS study reporting usability data.

43. Contreras CM, Metzger GA, Beane JD, Dedhia PH, Ejaz A, Pawlik TM. Telemedicine: patient-provider clinical engagement during the COVID-19 pandemic and beyond. J Gastrointestin Surg. 2020;24: 1692-7. https://doi.org/10.1007/s11605-020-04623-5.

44. Centers for Medicare \& Medicaid Services. Transforming clinical practice initiative. https://innovation.cms.gov/innovation-models/ transforming-clinical-practices.

45. American Hospital Association statement before the U.S. Senate Committee on Health, Education, Labor and Pensions regarding Americas health IT transformation: Translating the promise of electronic health records into better care. 2015. https://www.aha.org/ testimony/2015-03-17-aha-statement-re-americas-health-ittransformation. Accessed May 142020.

46. Moyer VA, USPSTF. Screening for primary hypertension in children and adolescents: U.S. preventive services task force recommendation statement. Pediatrics. 2013;132(5):907-14. https://doi. org/10.1542/peds.2013-2864.

47. Duncombe SL, Voss C, Harris KC. Oscillometric and auscultatory blood pressure measurement methods in children: a systematic review and meta-analysis. J Hypertens. 2017;35(2):213-24. https:// doi.org/10.1097/HJH.0000000000001178.

Publisher's Note Springer Nature remains neutral with regard to jurisdictional claims in published maps and institutional affiliations. 